\title{
Correction to: Continuous Infusion Versus Intermittent Bolus of Beta-Lactams in Critically Ill Patients with Respiratory Infections: A Systematic Review and Meta-analysis
}

\author{
Young R. Lee ${ }^{1}$ - Pamela D. Miller ${ }^{1}$ - Saeed K. Alzghari ${ }^{2} \cdot$ Delilah D. Blanco $^{3}$ • \\ Jackson D. Hager ${ }^{1} \cdot$ Kailey S. Kuntz ${ }^{1}$
}

Published online: 7 November 2017

(C) Springer International Publishing AG, part of Springer Nature 2017

\section{Correction to: Eur J Drug Metab Pharmacokinet https://doi.org/10.1007/s13318-017- 0439-5}

Unfortunately, $\geq$ was found missing between scores and 20 in conclusion section of the online published article. The original article was corrected.

The first sentence should read as:
CI of beta-lactam antibiotics is associated with better cure rates and higher \% $\%$ T $>$ MIC when administered to adult critically ill patients with respiratory infections, but may be most beneficial in severely ill patients with sepsis or APACHE II scores $\geq 20$ from more resistant Gramnegative bacterial infections.

The original article can be found online at https://doi.org/10.1007/ s13318-017-0439-5.

Young R. Lee

young.lee@ttuhsc.edu

Pamela D. Miller

pamela.miller@ttuhsc.edu

Saeed K. Alzghari

s.alzghari@gmail.com

Delilah D. Blanco

dblanco@tgh.org

Jackson D. Hager

jackson.hager@ttuhsc.edu

Kailey S. Kuntz

kailey.kuntz@ttuhsc.edu

1 Department of Pharmacy Practice, Texas Tech University Health Sciences Center School of Pharmacy, 1718 Pine Street, Abilene, TX 79601, USA

2 Gulfstream Genomics, LLC, 9301 N Central Expressway STE 335, Dallas, TX 75231, USA

3 Tampa General Hospital, 1 Tampa General Circle, Tampa, FL 33606, USA 